\title{
Nanoformulation of Premixing Propofol Lipid Emulsion and Fentanyl Citrate and Their Effects on Acute Toxicity, Sedation, and Analgesia
}

\author{
Wei Gao, ${ }^{1}$ Baoyong Sha, ${ }^{2}$ Zhe Fan, ${ }^{1}$ Yansheng Liu, ${ }^{3}$ and Xin Shen ${ }^{1}$ \\ ${ }^{1}$ Department of Anesthesiology, The First Affiliated Hospital of Xian Jiaotong University, Xian 710061, China \\ ${ }^{2}$ Institute of Basic Medical Science, Xi'an Medical University, Xian 710021, China \\ ${ }^{3}$ Electron Microscopy Room, Xian Jiaotong University Health Science Center, Xian 710061, China \\ Correspondence should be addressed to Baoyong Sha; shabaoyong@gmail.com and Xin Shen; shenxinmzk@126.com
}

Received 12 January 2016; Revised 18 March 2016; Accepted 20 March 2016

Academic Editor: Abdelwahab Omri

Copyright (C) 2016 Wei Gao et al. This is an open access article distributed under the Creative Commons Attribution License, which permits unrestricted use, distribution, and reproduction in any medium, provided the original work is properly cited.

\begin{abstract}
To illustrate the effects of premixing propofol lipid emulsion (PLE) and fentanyl citrate (FC) on acute toxicity, sedation, and analgesia, sixty female mice were randomly assigned to individual and premixed groups. The median lethal dose $\left(\mathrm{LD}_{50}\right)$, median effective dose $\left(\mathrm{ED}_{50}\right)$ of loss of righting reflex, and $\mathrm{ED}_{50}$ of tail flick test in both groups were determined using the up and down procedure (FC:PLE $=1 \mu \mathrm{g}: 2 \mathrm{mg}$ ). PLE was immediately administered to the mice from the individual group via the tail vein after injecting FC. By contrast, FC was mixed with PLE before injection from the premixed group. No significant differences in $\mathrm{LD}_{50}$, histopathological examination, and $\mathrm{ED}_{50}$ sedation value were found between the groups. However, $\mathrm{ED}_{50}$ of analgesia in the premixed group decreased to half of that in the individual group. Transmission electron microscopic observation revealed $\sim 10 \mathrm{~nm}$ fusiform particles at the juncture of 200-300 nm particles in the mixture of PLE and FC compared with the single PLE and its mixture with saline, which may be attributed to the structure of FC. In conclusion, premixing PLE and FC enhanced synergic analgesia twofold but did not influence toxicity and sedation compared with individual injections.
\end{abstract}

\section{Introduction}

Propofol (2,6-diisopropylphenol) is widely used to induce and maintain sedation during operation and intensive care. Propofol is an important clinical intravenous anesthetic that presents rapid action and short-term effects. Given its high hydrophobicity, propofol is injected in clinical settings as a lipid emulsion with a particle size of $200-259 \mathrm{~nm}$ [1]. Propofol lipid emulsion is commonly prepared as follows: $1 \%$ propofol, $10 \%$ soybean oil, $1.2 \%$ purified egg phospholipids (emulsifier), and $2.25 \%$ glycerol (tonicity-adjusting agent); sodium hydroxide is added to adjust the $\mathrm{pH}$. However, the large amount of soybean oil long-chain triglyceride increases the serum triglyceride level and induces hypertriglyceridemia [2]. Thus, a new type of propofol medium/long-chain lipid emulsion has been developed, in which half of the soybean oil long-chain triglyceride is replaced with medium-chain triglycerides to reduce the abovementioned risks [3].
Given its weak analgesic effect [4], propofol has been considered to be combined with an opioid analgesic, such as fentanyl, in clinical medicine. As a strong agonist of $\mu$ opioid receptors, fentanyl exhibits rapid action and shortterm efficacy. The aqueous solubilities of fentanyl and fentanyl citrate are 0.2 and $25 \mathrm{mg} / \mathrm{mL}$, respectively [5, 6]. Citrate modification reduces the hydrophobicity of fentanyl. Regardless of the formulation, the combination of fentanyl and propofol exhibits a natural synergy. Fentanyl reduces the first-pass pulmonary uptake of propofol, and propofol inhibits the oxidative metabolism of opioid through the cytochrome P450 enzyme [7-9]. Neither of these drugs can be injected without hydrophilic formulations. Trissel et al. [10] reported that hydrophilic formulations of fentanyl and propofol that are mixed in a polypropylene syringe are physically compatible and chemically stable. They are commonly premixed by Ysite administration in clinical settings. A similar premixture, the single-syringe combination of ketamine and propofol, 
shows minimal adverse hemodynamic effects and favorable emergence characteristics for short procedures [11]. Premixing propofol with lidocaine is an effective measure to reduce injection pain $[12,13]$. However, the effects of premixing propofol lipid emulsion and fentanyl citrate on acute toxicity, sedation, and analgesia remain unknown.

In this study, the median lethal dose $\left(\mathrm{LD}_{50}\right)$ and median effective dose $\left(\mathrm{ED}_{50}\right)$ of premixed and individual solutions of propofol lipid emulsion and fentanyl citrate were determined in mice. Hematoxylin and eosin- (HE-) stained pathological sections of important organs in the $\mathrm{LD}_{50}$ experiment were obtained. Transmission electron micrographs (TEM) of propofol lipid emulsion and/or fentanyl citrate were analyzed to elucidate the potential mechanism of the interaction between propofol lipid emulsion and fentanyl citrate.

\section{Materials and Methods}

2.1. Animals. A total of 60 female Kunming mice weighing 18-22 g were obtained from the Laboratory Animal Research Center of Xian Jiaotong University. All animal experiments were performed in accordance with the European Commission Directive 86/609/EEC (European Convention for the Protection of Vertebrate Animals Used for Experimental and Other Scientific Purposes) and the Guiding Principles in the Use of Animals in Toxicology, and these experiments were approved by the Xian Jiaotong University Ethics Committee. Animal suffering was minimized. All mice were maintained in an animal house with a $12 \mathrm{~h}$ light/12 $\mathrm{h}$ dark cycle under $22 \pm 2^{\circ} \mathrm{C}$ and $55 \% \pm 5 \%$ humidity. Sterilized food pellets and water were provided ad libitum.

2.2. Drug Administration. The propofol medium/long-chain lipid emulsion and fentanyl citrate were purchased from Fresenius Kabi Co., Ltd. (Beijing. China) and Yichang Humanwell Pharmaceutical Co., Ltd. (Yichang, China), respectively. The carbon support film on copper was purchased from Beijing Zhongjingkeyi Technology Co., Ltd. (Beijing, China).

All 60 mice were randomly assigned to individual and premixed groups for each test. Each group in each test was administered with different doses of fentanyl and propofol. The dosage ratio of fentanyl citrate and propofol lipid emulsion was 1:2000 in this study. Propofol lipid emulsion was immediately administered in the mice from the individual group via the tail vein after injecting fentanyl citrate. By contrast, fentanyl citrate was mixed with propofol lipid emulsion before tail vein injection in the mice from the premixed group. $\mathrm{LD}_{50}$ and $\mathrm{ED}_{50}$ were determined using the up and down procedure in accordance with the OECD Guidelines for Testing of Chemicals Number 425 (OECD, 2008) on female Kunming mice [14-16].

2.3. Determination of $L D_{50}$. In the limit test for each group, a single limit dose of $54.40 \mathrm{mg} / \mathrm{kg}$ propofol $+27.20 \mu \mathrm{g} / \mathrm{kg}$ fentanyl was estimated and intravenously administered [17]. The electrocardiograms of all mice were monitored for 30 min using a BIOPAC system (BIOPAC Systems Inc., Goleta, CA, USA) to determine death case. A constant straight line from the electrocardiograms was regarded as the death criterion. Histopathological examinations through HE staining were performed on the brain, lung, heart, liver, and kidney of a dead mouse within $1 \mathrm{~h}$ after death from the limit dose administered. In the main test for each group, one female mouse was randomly selected and intravenously administered with a preliminary dose of $17.84 \mathrm{mg} / \mathrm{kg}$ propofol $+8.92 \mu \mathrm{g} / \mathrm{kg}$ fentanyl. Single animals were dosed in sequence at $1 \mathrm{~h}$ intervals for the rapid cardiovascular and respiratory depression of propofol $[18,19]$. The subsequent ascending or descending doses were determined depending on the survival from the preceding dose of the animals with a dose progression factor of 1.25 .

2.4. Righting Reflex Test. The up and down procedure was used in the loss of righting reflex (LRR) assay to determine $\mathrm{ED}_{50}$ of sedation [20]. The LRR for more than $10 \mathrm{~s}$ was regarded as positive for sedation; otherwise, the observation was regarded as negative [21]. For the limit test for each group, a single limit dose of $9.13 \mathrm{mg} / \mathrm{kg}$ propofol $+4.57 \mu \mathrm{g} / \mathrm{kg}$ fentanyl was estimated and intravenously administered [17]. In the main test for each group, one female mouse was randomly selected and intravenously administered with a preliminary dose of $2.39 \mathrm{mg} / \mathrm{kg}$ propofol $+1.20 \mu \mathrm{g} / \mathrm{kg}$ fentanyl. The subsequent ascending or descending doses were determined depending on the results of LRR from the preceding dose of the animals with a dose progression factor of 1.25 .

2.5. Tail Flick Test. The tail flick test was used to estimate $\mathrm{ED}_{50}$ of analgesia. The tail of each mouse was immersed in hot water at $55 \pm 0.5^{\circ} \mathrm{C}$. The tail flick latency was defined as the time interval taken by mice to flick their tails after heat exposure. Cutoff time was fixed at $30 \mathrm{~s}$ to avoid damage to the animal tails. The increase in tail flick latency was taken as antinociception and calculated as the percentage of maximal possible effect (MPE) by using the following formula: $\mathrm{MPE} \%=\left(t_{1}-t_{c}\right) /\left(t_{\text {cutoff }}-t_{c}\right) \times 100$, where $t_{1}$ is the withdrawal latency, $t_{c}$ is the control latency, and $t_{\text {cutoff }}$ is the cutoff time [22]. The up and down method is intended for quantal data; thus, the graded tail flick data were converted to a quantal scale with a response of at least 50\% MPE defined as analgesic and a value less than 50\% MPE defined as not analgesic [23]. In the limit test for each group, a single limit dose of $9.13 \mathrm{mg} / \mathrm{kg}$ propofol $+4.57 \mu \mathrm{g} / \mathrm{kg}$ fentanyl was estimated and intravenously administered [22]. In the main test for each group, one female mouse was randomly selected and intravenously administered with a preliminary dose of $2.39 \mathrm{mg} / \mathrm{kg}$ propofol $+1.20 \mu \mathrm{g} / \mathrm{kg}$ fentanyl. The following ascending or descending doses were determined depending on the results of MPE\% from the preceding dose of the animals with a dose progression factor of 1.25.

2.6. Morphology of Propofol Lipid Emulsion and Fentanyl Citrate. The morphology of propofol lipid emulsion and fentanyl citrate was investigated via TEM (Hitachi, Japan) [24]. The samples included single fentanyl citrate $(50 \mu \mathrm{g} / \mathrm{mL})$, single propofol lipid emulsion $(10 \mathrm{mg} / \mathrm{mL})$, mixed propofol 
TABLE 1: Acute toxicity, sedation, and analgesia test in the individual and premixed groups of propofol lipid emulsion and fentanyl citrate.

\begin{tabular}{llcccccc}
\hline & & \multicolumn{2}{c}{ Acute toxicity test } & \multicolumn{2}{c}{ Sedation test } & \multicolumn{2}{c}{ Analgesia test } \\
& & $\mathrm{LD}_{50}$ & $\mathrm{CI} 95 \%$ & $\mathrm{ED}_{50}$ & $\mathrm{CI}_{95 \%}$ & $\mathrm{ED}_{50}$ & $\mathrm{CI}_{95 \%}$ \\
\hline \multirow{2}{*}{ Individual group } & Propofol $(\mathrm{mg} / \mathrm{kg})$ & 34.84 & $27.68,43.30$ & 7.31 & $5.98,12.30$ & 7.30 & $5.85,7.31$ \\
& Fentanyl $(\mu \mathrm{g} / \mathrm{kg})$ & 17.42 & $13.84,21.65$ & 3.36 & $2.99,6.15$ & 3.65 & $2.93,3.66$ \\
\hline \multirow{2}{*}{ Premixed group } & Propofol $(\mathrm{mg} / \mathrm{kg})$ & 34.84 & $29.46,47.00$ & 5.85 & $4.78,9.94$ & $3.74^{*}$ & $2.95,4.69$ \\
& Fentanyl $(\mu \mathrm{g} / \mathrm{kg})$ & 17.42 & $14.73,23.50$ & 2.93 & $2.39,4.97$ & $1.87^{*}$ & $1.48,2.19$ \\
\hline
\end{tabular}

Median lethal dose $\left(\mathrm{LD}_{50}\right)$, median effective dose $\left(\mathrm{ED}_{50}\right)$, and $95 \%$ confidence interval $(\mathrm{CI} 95 \%) .{ }^{*} \mathrm{P}<0.05$ between groups.

lipid emulsion and fentanyl citrate (volume ratios of $20: 1$, $20: 2,20: 3,20: 4$, and $20: 5$ ), and mixed propofol and physiological saline (volume ratios of $20: 2$ and $20: 5$ ). The images were captured immediately after mixing. Samples were dropped on a copper grid, air-dried, and then stained with 3\% phosphotungstic acid for TEM. To reduce the bias, TEM was operated by an experienced electron microscope technician who was blinded to the study design. The center area of copper grid was first located at low magnification $(10000 \mathrm{x})$, and the nearest nanoparticle gathered grids on four directions were visualized at low and high (50000x) magnifications under a relatively low electron current density. Eight TEM images were obtained for each sample to obtain the emulsion overview.

2.7. Statistical Analysis. The $\mathrm{LD}_{50}$ and $\mathrm{ED}_{50}$ values, as well as the $95 \%$ confidence interval (CI), were analyzed using the AOT 425 Statistical Program. $\mathrm{ED}_{50}$ values with nonoverlapping 95\% CIs were considered significantly different.

\section{Results}

3.1. $L D_{50}$ from the Up and Down Procedure. In the limit test, a female mouse in both groups died after intravenous administration of a single dose of $54.40 \mathrm{mg} / \mathrm{kg}$ propofol + $27.20 \mu \mathrm{g} / \mathrm{kg}$ fentanyl. HE-stained examinations showed no significant abnormal changes in the brain, lung, heart, liver, and kidney in both groups after the limit dose (Figure 1). The results of the respective up and down test series in both groups in the main test were shown in Figure 2. The estimated $\mathrm{LD}_{50}$ in the premixed group was $34.84 \mathrm{mg} / \mathrm{kg}$ propofol combined with $17.42 \mu \mathrm{g} / \mathrm{kg}$ fentanyl, and $\mathrm{LD}_{50}$ in the individual group was $34.84 \mathrm{mg} / \mathrm{kg}$ propofol $+17.42 \mu \mathrm{g} / \mathrm{kg}$ fentanyl, which showed no significant difference between the two groups (Table 1).

3.2. Righting Reflex. The sequences of the up and down method for the righting reflex test in both groups are shown in Figure 3. The $\mathrm{ED}_{50}$ value of sedation in the premixed groups was $5.85 \mathrm{mg} / \mathrm{kg}$ propofol combined with $2.93 \mu \mathrm{g} / \mathrm{kg}$ fentanyl. The corresponding $\mathrm{ED}_{50}$ in the individual groups was $7.31 \mathrm{mg} / \mathrm{kg}$ propofol combined with $3.66 \mu \mathrm{g} / \mathrm{kg}$ fentanyl. No significant difference in $\mathrm{ED}_{50}$ of LRR was detected between the individual and premixed groups (Table 1).

3.3. Tail Flick Test. The results of the respective up and down test series in both groups in the tail flick test of analgesia were shown in Figure $4 . \mathrm{ED}_{50}$ of analgesia in the individual groups was $7.30 \mathrm{mg} / \mathrm{kg}$ propofol combined with $3.65 \mu \mathrm{g} / \mathrm{kg}$ fentanyl. The corresponding $\mathrm{ED}_{50}$ in the premixed group was $3.74 \mathrm{mg} / \mathrm{kg}$ propofol combined with $1.87 \mu \mathrm{g} / \mathrm{kg}$ fentanyl. The $\mathrm{ED}_{50}$ value of the premixed group was significantly lower than that of the individual group (Table 1).

\subsection{Morphology of Propofol Lipid Emulsion and Fentanyl} Citrate. As shown in Figure 5, large particles $(>1 \mu \mathrm{m})$ were absent in all TEM micrographs. The propofol lipid emulsion had a wide inhomogeneous particle diameter (200-300 nm) with a large-area solution field (Figure 5(a)). The particle size in all mixtures of propofol lipid emulsion and fentanyl citrate injection (Figures 5(b)-5(f)) and mixtures of propofol and physiological saline (volume ratio of $20: 2$ ) (Figure 5(g)) had no visible difference compared with that in the propofol lipid emulsion. However, the distribution area and numbers of particles in one visual field were greater in the mixtures than in the propofol lipid emulsion. Furthermore, $\sim 10 \mathrm{~nm}$ fusiform particles were found at the juncture of the 200$300 \mathrm{~nm}$ particles in all propofol lipid emulsion and fentanyl citrate mixtures. However, these particles were absent in the single propofol lipid emulsion and in its mixture with physiological saline. In addition, the mixture of propofol and physiological saline at a volume ratio of $20: 5$ (Figure $5(\mathrm{~h})$ ) contained $40-60 \mathrm{~nm}$ particles. A TEM image of the black dried salt crystal of fentanyl citrate under vacuum was shown in Figure 5(i).

\section{Discussion}

Propofol and fentanyl are hydrophobic drugs. Their small molecular size and hydrophobic nature allow them to penetrate the blood cerebrospinal barrier and rapidly exert sedative and analgesic effects [25]. However, hydrophobic drugs cannot be directly injected. They must first be prepared into several types of hydrophilic formulations, such as molecular complexes, polymeric micellar systems, nanosuspensions, lipid formulations, and prodrugs, before they can be used [26]. In current clinical settings, propofol is prepared into propofol lipid emulsion, whereas fentanyl is prepared into fentanyl citrate. Propofol lipid emulsion and fentanyl citrate are hydrophilic formulations of hydrophobic drugs that are frequently injected separately and sequentially for anesthesia. Few reports focused on the premixture of fentanyl citrate and propofol lipid emulsion. The fentanyl citrate analog alfentanil hydrochloridum can be premixed with propofol 


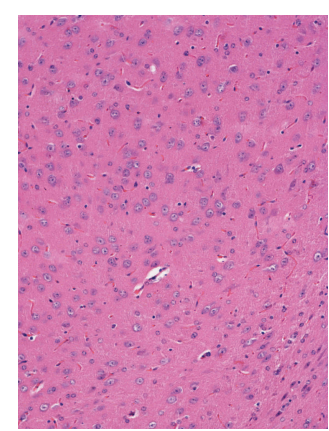

(a)

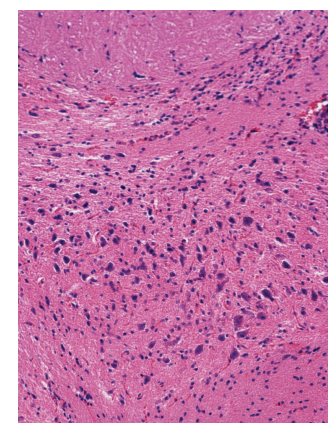

(f)

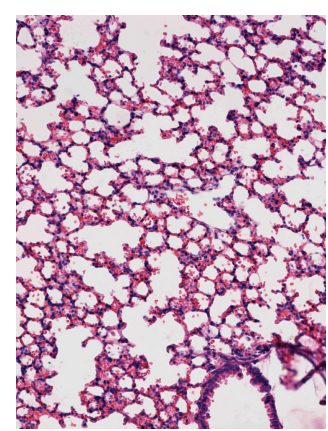

(b)

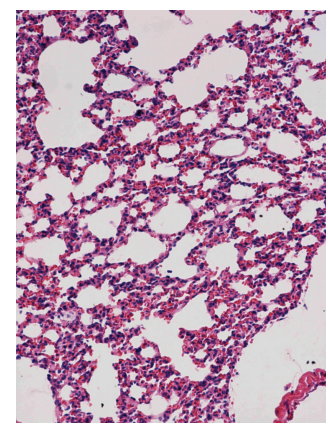

(g)

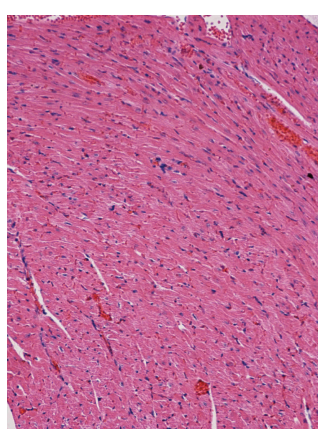

(c)

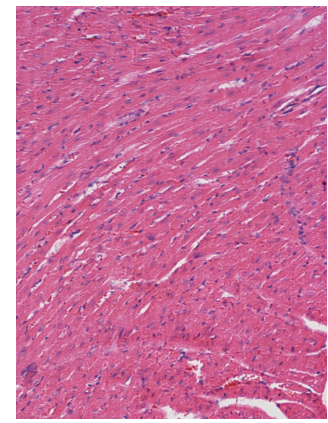

(h)

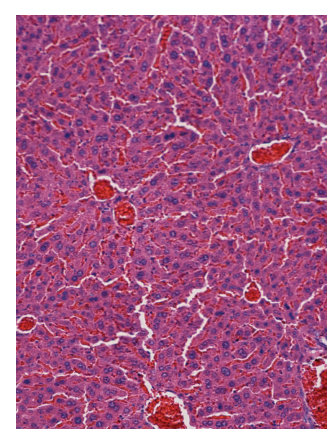

(d)

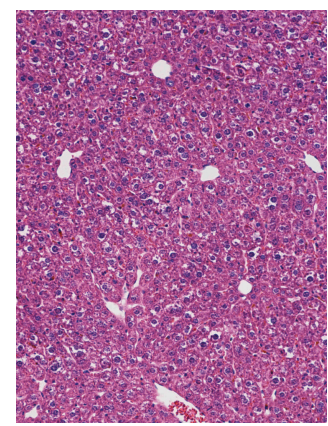

(i)

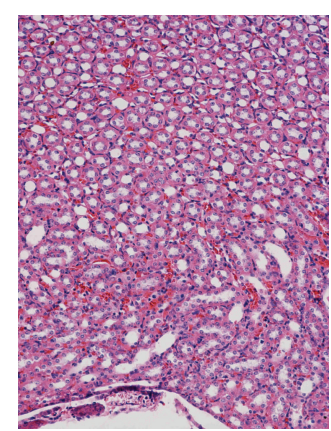

(e)

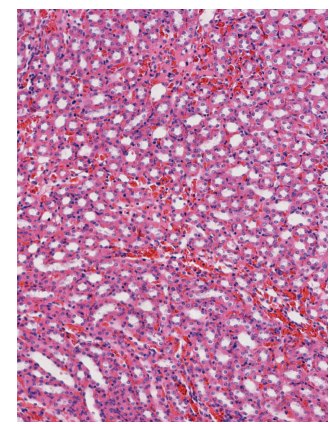

(j)

FIGURE 1: HE-stained pathological sections of organs at the highest administered dose $(54.40 \mathrm{mg} / \mathrm{kg}$ propofol lipid emulsion combined with $27.20 \mu \mathrm{g} / \mathrm{kg}$ fentanyl citrate) (200x). (a-e) Pathological sections of the brain, lung, heart, liver, and kidney in the individual group. (f-j) Pathological sections of the brain, lung, heart, liver, and kidney in the premixed group.

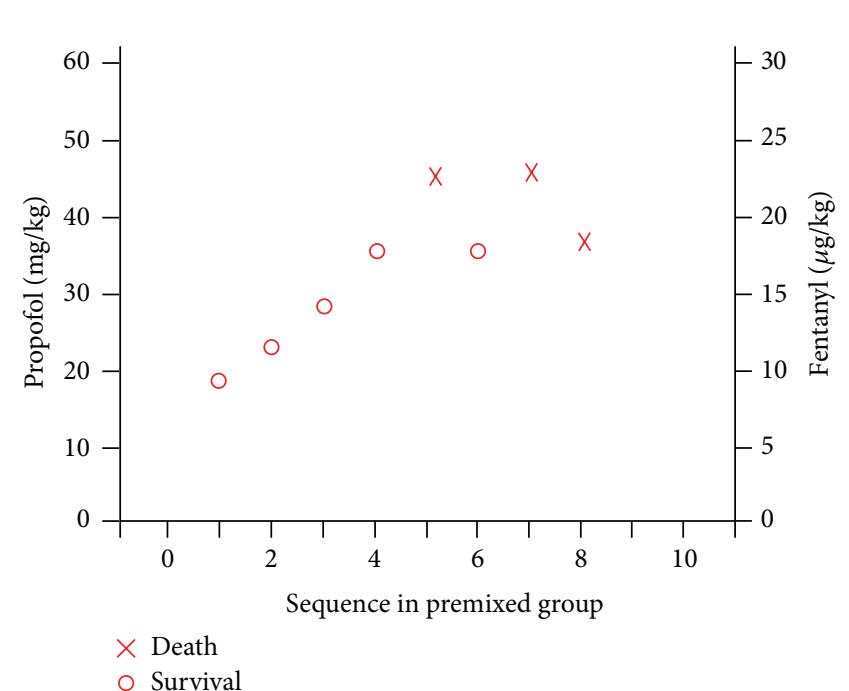

(a)

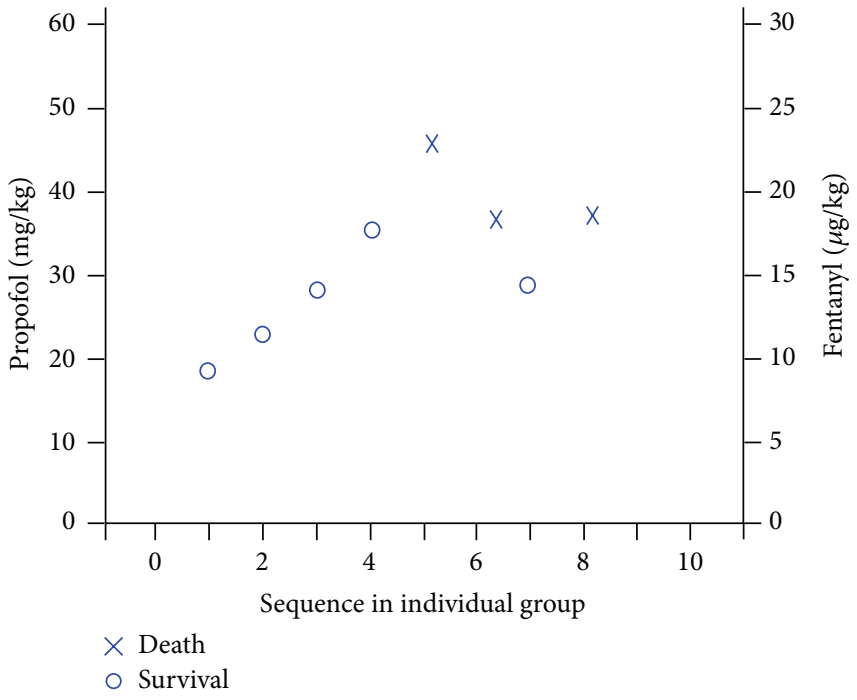

(b)

FIGURE 2: Sequence of the up and down procedure for $\mathrm{LD}_{50}$. (a) Premixed group. (b) Individual group.

lipid emulsion at a ratio of $20: 1$ to $50: 1$ for use within $6 \mathrm{~h}$ of preparation, thereby implying the feasibility of preparing a premixture for fentanyl citrate and propofol lipid emulsion [13]. In consideration that such a premixing administration method is not contained in drug instructions, the premixed solution of propofol lipid emulsion and fentanyl citrate should be preliminarily investigated in animals.
In this study, no significant differences in $\mathrm{LD}_{50}$ values and HE-stained histopathological examinations were found between the premixed and individual groups (Table 1, Figure 1). The premixture of fentanyl citrate and propofol lipid emulsion showed no increased toxicity. Similarly, the $\mathrm{ED}_{50}$ value of sedation in the premixed group showed no significant difference compared with that in the individual group. 


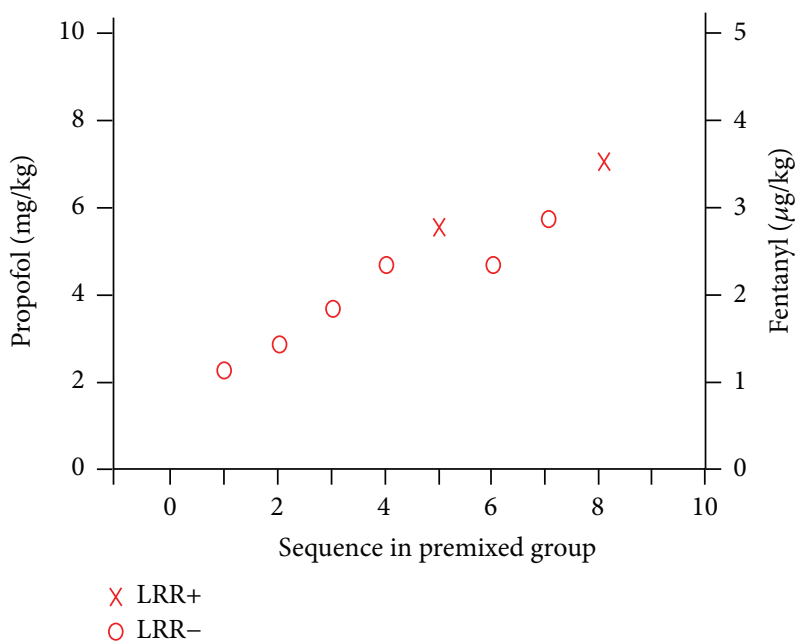

(a)

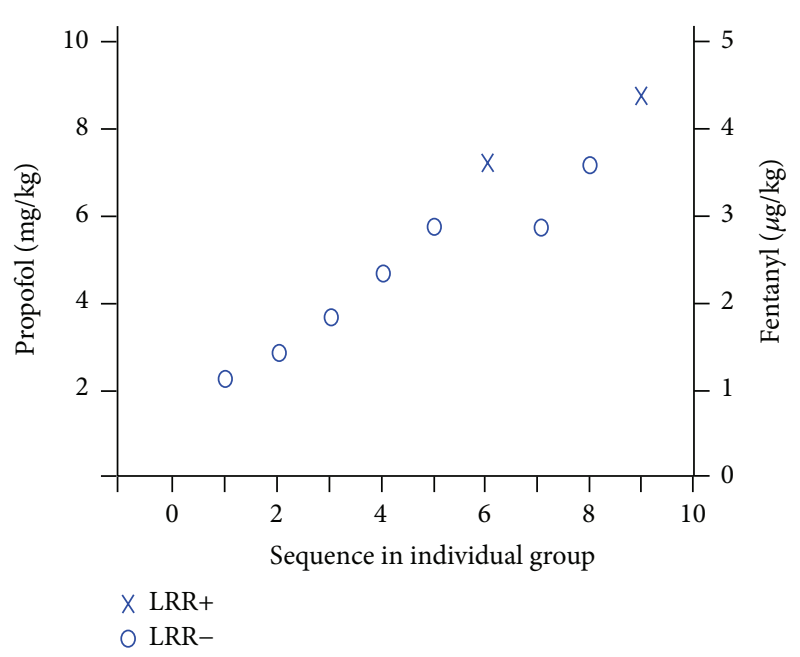

(b)

Figure 3: Sequences of the up and down procedure for $\mathrm{ED}_{50}$ of sedation. (a) Premixed group. (b) Individual group.

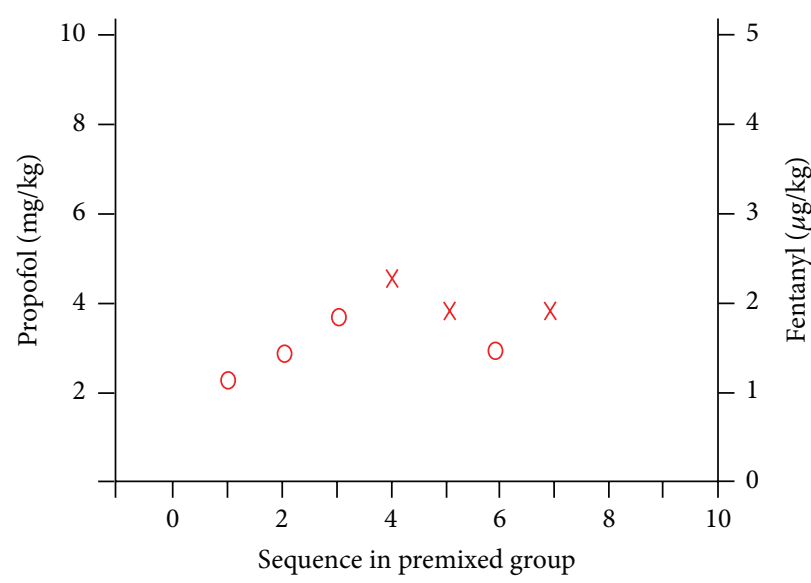

$X \geq 50 \%$ MPE

$0<50 \%$ MPE

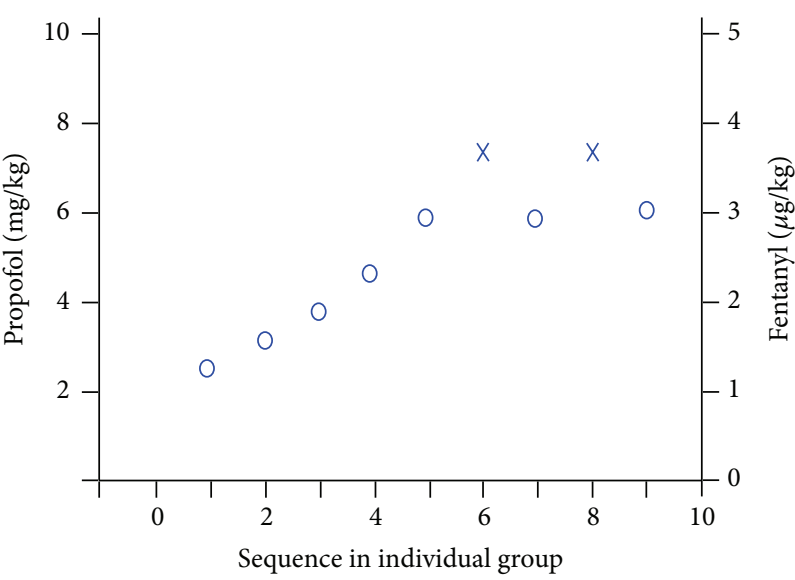

$X \geq 50 \% \mathrm{MPE}$

$0<50 \%$ MPE

(a)

(b)

FIgURE 4: Sequences of the up and down procedure for $\mathrm{ED}_{50}$ of analgesia. (a) Premixed group. (b) Individual group.

This result indicates that the prior mixture did not change the synergistic effect of propofol on sedation. However, the $\mathrm{ED}_{50}$ value of analgesia in the premixed group was significantly lower than that in the individual group, demonstrating that the prior mixture enhanced the synergistic effect of fentanyl on analgesia. Thus, the animal experiment revealed that the synergistic analgesic effect was stronger in the premixed group than in the individual group. No significant differences in toxicity and synergic sedation were found between the two groups. Similar results have been reported in other literature. A single shot of propofol and rocuronium shows that the potency of rocuronium is significantly enhanced after propofol infusion for 30 min compared with 2 min of propofol; this finding indicates that propofol can increase the affinity of rocuronium for the receptor under steady-state conditions [27]. Meanwhile, a randomized double-blinded trial found no statistically significant difference in the doses of propofol required for inducing anesthesia, regardless of whether the drug was administered as a freshly prepared propofol/lidocaine 10:1 mixture or as a separate injection after a dose of lidocaine [28].

The TEM micrographs of propofol lipid emulsion and fentanyl citrate or physiological saline with different volume ratios were examined to elucidate further the results of the animal experiment. Lipid emulsion is an unstable oil in water systems, and the intravenous administration of droplets ( $>5 \mu \mathrm{m}$ in diameter) may pose the risk of pulmonary embolism [29]. For example, adding lidocaine to propofol emulsion in one or separated syringe is widely recommended to minimize injection pain $[12,30]$. However, Masaki et al. [31] added $2 \mathrm{~mL}$ of $2 \%$ lidocaine to $20 \mathrm{~mL}$ of $1 \%$ propofol and observed particle sizes higher than $5 \mu \mathrm{m}$ in scanning electron 


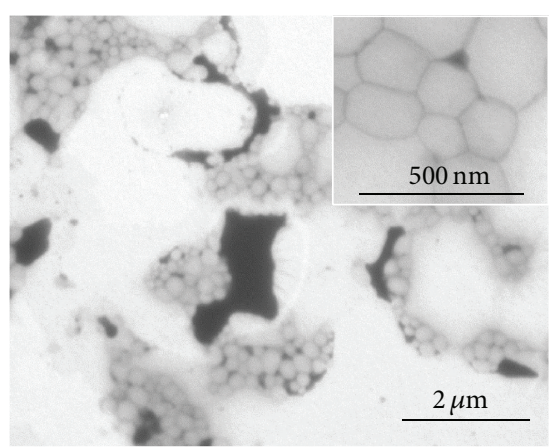

(a)

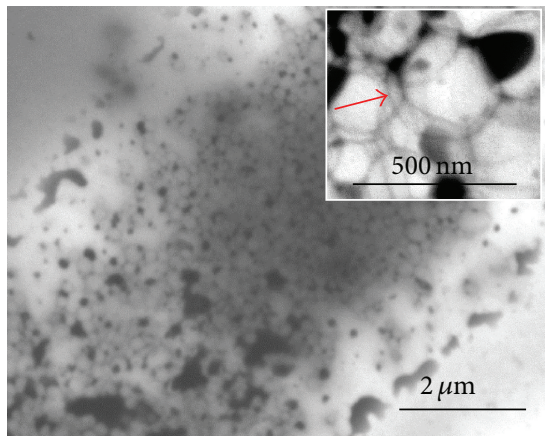

(d)

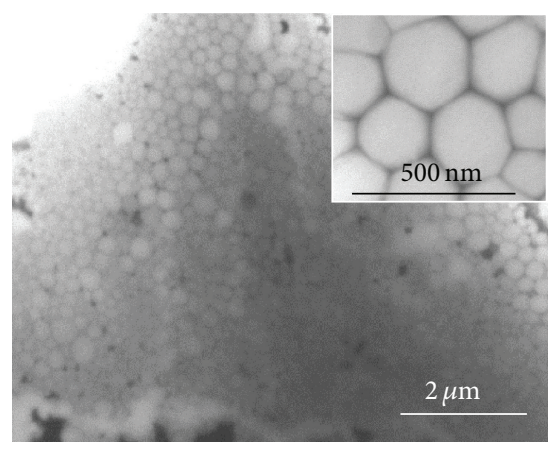

(g)

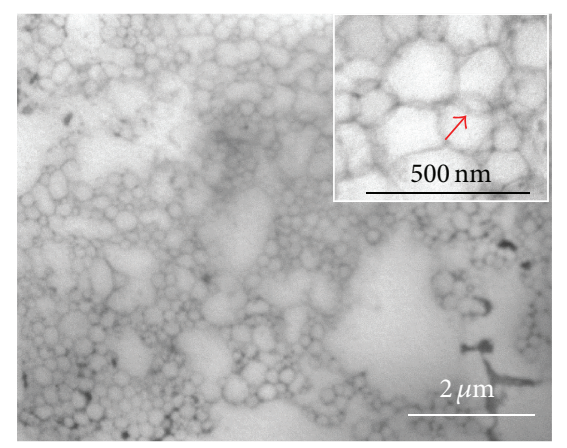

(b)

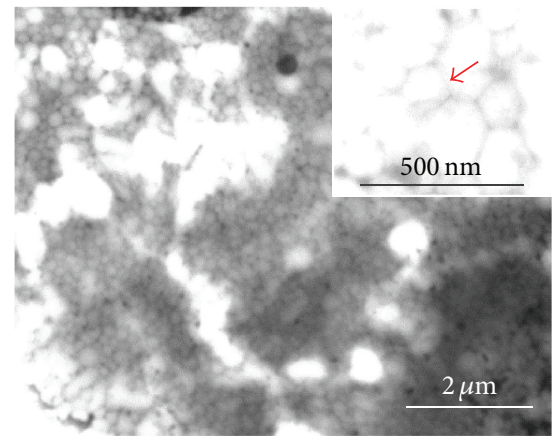

(e)

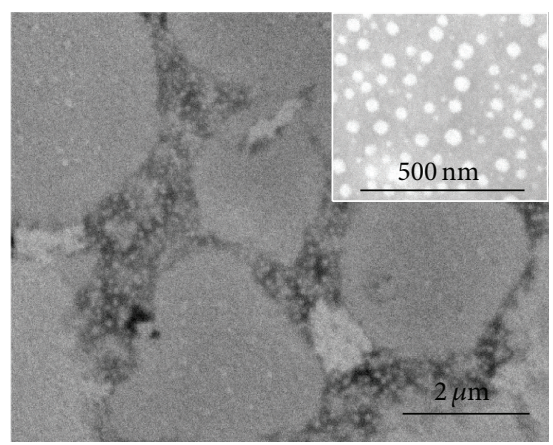

(h)

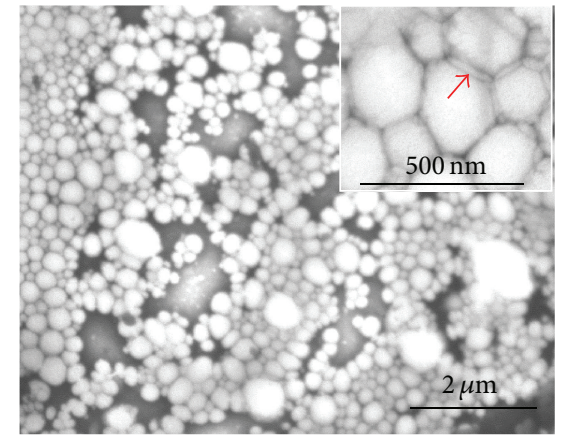

(c)

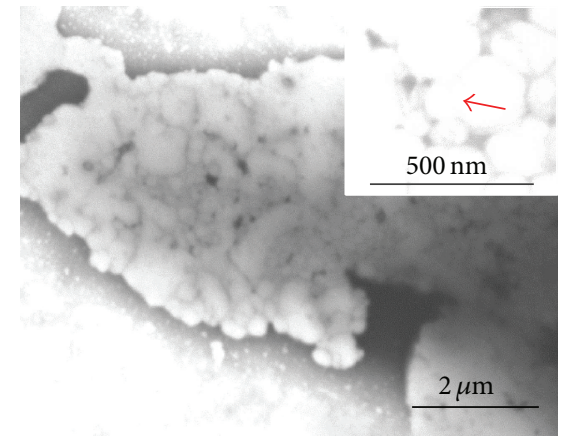

(f)

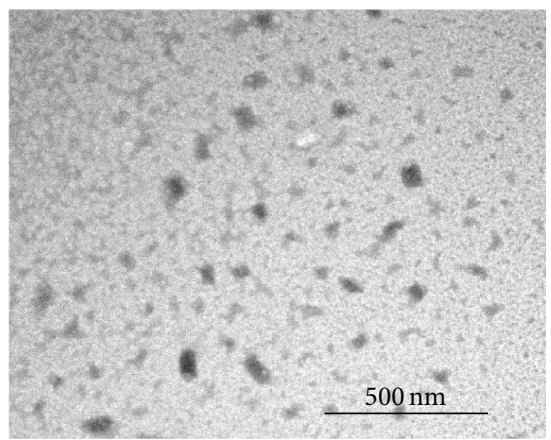

(i)

FIGURE 5: TEM micrographs of propofol lipid emulsion, fentanyl citrate, mixture of propofol lipid emulsion and fentanyl citrate, and mixture of propofol lipid emulsion and physiological saline. (a) Propofol lipid emulsion. (b-f) Mixed propofol lipid emulsion and fentanyl citrate (volume ratios of $20: 1,20: 2,20: 3,20: 4$, and $20: 5$ ). The arrow pointed to $\sim 10 \mathrm{~nm}$ fusiform particles at the juncture of 200-300 nm particles. (g-h) Mixed propofol and physiological saline (volume ratios of $20: 2$ and $20: 5$ ). (i) Fentanyl citrate.

micrographs. Park et al. [32] reported that the particle size increases to $>5 \mu \mathrm{m}$ at $2 \mathrm{~h}$ after adding $>30 \mathrm{mg}$ of $4 \%$ lidocaine. Thus, the maximum volume dilution rate of propofol with $1 \%$ lidocaine is $20: 1$ in the drug instruction of propofol lipid emulsion and is injected immediately after premixing. In the present study, the particles were $<1 \mu \mathrm{m}$ after TEM analysis, indicating that the premixture did not significantly increase the size of propofol lipid emulsion and the risk of pulmonary embolism (Figure 5). In consideration that fentanyl and propofol are commonly combined and used during the induction period, the images were captured immediately after premixing. The absence of particles $>5 \mu \mathrm{m}$ might explain why the $\mathrm{LD}_{50}$ values and HE-stained histopathological test results did not significantly change in these two administration methods. Furthermore, the cardiovascular or respiratory depression might be the main reason for death $[18,19]$. The intravenous injection $\mathrm{LD}_{50}$ values of propofol and fentanyl were 53 and $11.2 \mathrm{mg} / \mathrm{kg}$ in mice according to the drug instructions, respectively. The dosage ratio of fentanyl citrate and propofol lipid emulsion was 1:2000 in this study. Thus, the death was mainly induced by propofol not fentanyl.

The large-area solution field in the propofol lipid emulsion was probably caused by the instability of the lipid emulsion system (Figure 5(a)). Lipid emulsion degrades because of Brownian motion or external agitation, which occurs in several sequential processes, including dispersed phase, flocculation, coalescence, and free oil [33, 34]. Although the morphology observed via TEM is not totally 
equal to it in emulsion, TEM is an important method to observe nanosized lipid emulsions [24]. A large amount of free propofol leaked from the particles and dissolved in the oil phase of the propofol lipid emulsion. Particle size did not significantly change with increasing fentanyl citrate concentration in the mixture of propofol emulsion and fentanyl citrate injection (Figures 5(b)-5(f)). However, the particle number in these mixtures was greater than that in pure propofol lipid emulsion, and the solution field areas in these mixtures were smaller than those in the single propofol lipid emulsion. These data illustrate that fentanyl citrate injection increased the number of particles and reduced the free propofol from the propofol emulsion. Compared with the mixture of propofol and physiological saline at a $20: 2$ ratio (Figure $5(\mathrm{~g})$ ), the mixture of propofol lipid emulsion and fentanyl citrate at a 20:2 ratio showed $\sim 10 \mathrm{~nm}$ fusiform particles at the juncture of large particles $(200-300 \mathrm{~nm})$ (Figure 5(c)). Compared with the mixture of propofol lipid emulsion and physiological saline at a $20: 5$ ratio (Figure $5(\mathrm{~h})$ ), the mixture of propofol lipid emulsion and fentanyl citrate at a 20:5 ratio produced significantly larger particles $(200-300 \mathrm{~nm})$ surrounded by $\sim 10 \mathrm{~nm}$ fusiform particles (Figure 5(f)). These results suggest that the water/oil ratio played a negligible role in the change of the propofol lipid emulsion and fentanyl citrate mixture. Thus, the structure of fentanyl citrate should be considered. Fentanyl citrate is an organic basic compound with a $\mathrm{p} K_{\mathrm{a}}$ value of 8.99 [35]. Fentanyl citrate has a hydrophobic part on one side and a hydrophilic part on the other side, with a positive charge at physiological pH 7.35-7.45 and a structure similar to that of a cationic surfactant [36]. Fentanyl citrate is a cationic surfactant located at the edge of the particle for the amphipathicity and electrostatic adsorption with the negative-charged emulsifier of egg phospholipid. These characteristics might explain the larger number of $200-300 \mathrm{~nm}$ particles that did not significantly change for water/oil ratio and the $\sim 10 \mathrm{~nm}$ fusiform particles at the juncture of $200-300 \mathrm{~nm}$ particles in the mixture of propofol lipid emulsion and fentanyl citrate. Meanwhile, fentanyl might dissociate from citrate upon sodium hydroxide or dilution in the propofol lipid emulsion because of the instability of ionic bonds in fentanyl citrate and then dissolve in the soybean oil of lipid emulsion [37]. Such a high hydrophobicity allows fentanyl to penetrate the blood cerebrospinal barrier [38]. However, TEM only showed morphological changes, and the reasons for enhanced synergic analgesia were deduced by chemical theory, which needs further research to validate.

\section{Conclusion}

Compared with individual injections, the premixed solution of propofol lipid emulsion and fentanyl citrate at a volume ratio of $20: 2$ enhanced synergic analgesia twofold but did not influence acute toxicity and sedation. These results indicated that formulation interaction played an important role in the drug interaction. In clinical settings, not only the characteristics of drugs themselves but also their formulation should be considered. A rational administration would improve the effects of combined drugs.

\section{Competing Interests}

The authors declare no competing financial interests.

\section{Authors' Contributions}

Wei Gao and Baoyong Sha contributed equally to this work.

\section{Acknowledgments}

This study was supported in part by the National Nature Science Foundation of China (81301308, 81301040), Natural Science Basic Research Plan in Shaanxi Province of China (2014JQ4158), Program for Youth Science and Technology Star of Shaanxi Province (2014KJXX-76), and Doctoral Starting Foundation of Xi'an Medical University (2015DOC11), 2014 Chinese Anesthesiologist Association young talented anesthesiologist abroad training grant, and Young Scholar Research Grant of Chinese Anesthesiologist Association (220150800002).

\section{References}

[1] M. T. Baker and M. Naguib, "Propofol: The challenges of formulation," Anesthesiology, vol. 103, no. 4, pp. 860-876, 2005.

[2] W. Jiang, Z.-B. Yang, Q.-H. Zhou, X. Huan, and L. Wang, "Lipid metabolism disturbances and AMPK activation in prolonged propofol-sedated rabbits under mechanical ventilation," Acta Pharmacologica Sinica, vol. 33, no. 1, pp. 27-33, 2012.

[3] H. J. Theilen, S. Adam, M. D. Albrecht, and M. Ragaller, "Propofol in a medium- and long-chain triglyceride emulsion: pharmacological characteristics and potential beneficial effects," Anesthesia and Analgesia, vol. 95, no. 4, pp. 923-929, 2002.

[4] I. Vasileiou, T. Xanthos, E. Koudouna et al., "Propofol: a review of its non-anaesthetic effects," European Journal of Pharmacology, vol. 605, no. 1-3, pp. 1-8, 2009.

[5] M. J. O’Neil, The Merck Index: An Encyclopedia of Chemicals, Drugs, and Biologicals, The Royal Society of Chemistry, 15th edition, 2013.

[6] S. D. Roy and G. L. Flynn, "Solubility and related physicochemical properties of narcotic analgesics," Pharmaceutical Research, vol. 5, no. 9, pp. 580-586, 1988.

[7] J. F. A. Hendrickx, E. I. Eger, J. M. Sonner, and S. L. Shafer, "Is synergy the rule? A review of anesthetic interactions producing hypnosis and immobility," Anesthesia and Analgesia, vol. 107, no. 2, pp. 494-506, 2008.

[8] J. Vuyk, "Pharmacokinetic and pharmacodynamic interactions between opioids and propofol," Journal of Clinical Anesthesia, vol. 9, no. 6, supplement 1, pp. 23S-26S, 1997.

[9] J. A. Kuipers, F. Boer, W. Olieman, A. G. L. Burm, and J. G. Bovill, "First-pass lung uptake and pulmonary clearance of propofol: assessment with a recirculatory indocyanine green pharmacokinetic model," Anesthesiology, vol. 91, no. 6, pp. 17801787, 1999.

[10] L. A. Trissel, D. L. Gilbert, and J. F. Martinez, "Compatibility of propofol injectable emulsion with selected drugs during simulated Y-site administration," American Journal of HealthSystem Pharmacy, vol. 54, no. 11, pp. 1287-1292, 1997. 
[11] R. F. Grace, W. Tang, and E. Namel, "An audit of the haemodynamic and emergence characteristics of single-shot 'ketofol," Anaesthesia and Intensive Care, vol. 43, no. 4, pp. 503-505, 2015.

[12] B. Bachmann-Mennenga, A. Ohlmer, R. H. Boedeker, M. Mann, B. Mühlenbruch, and M. Heesen, "Preventing pain during injection of propofol: effects of a new emulsion with lidocaine addition," European Journal of Anaesthesiology, vol. 24, no. 1, pp. 33-38, 2007.

[13] T. E. Healy and P. R. Knight, Wylie Churchill-Davidson's A Practice of Anesthesia, Taylor \& Francis, 7th edition, 2003.

[14] Q. Zhao, M. Yang, Y. Deng et al., "The safety evaluation of salvianolic acid B and ginsenoside Rg1 combination on mice," International Journal of Molecular Sciences, vol. 16, no. 12, pp. 29345-29356, 2015.

[15] J. Buschmann, "The OECD guidelines for the testing of chemicals and pesticides," Methods in Molecular Biology, vol. 947, pp. 37-56, 2013.

[16] A. Rispin, D. Farrar, E. Margosches et al., "Alternative methods for the median lethal dose (LD50) test: the up-and-down procedure for acute oral toxicity," ILAR Journal, vol. 43, no. 4, pp. 233-243, 2002.

[17] Y. Zhou, J. Yang, J. Liu, Y. Wang, and W. S. Zhang, "Efficacy comparison of the novel water-soluble propofol prodrug HX0969w and fospropofol in mice and rats," British Journal of Anaesthesia, vol. 111, no. 5, pp. 825-832, 2013.

[18] M. Kashiwagi, Y. Okada, S.-I. Kuwana, S. Sakuraba, R. Ochiai, and J. Takeda, "Mechanism of propofol-induced central respiratory depression in neonatal rats: anatomical sites and receptor types of action," Advances in Experimental Medicine and Biology, vol. 551, pp. 221-226, 2004.

[19] T. Shintaku, T. Ohba, H. Niwa et al., "Effects of propofol on electrocardiogram measures in mice," Journal of Pharmacological Sciences, vol. 126, no. 4, pp. 351-358, 2014.

[20] R. A. Whitehead, S. K. Schwarz, Y. I. Asiri, T. Fung, E. Puil, and B. A. MacLeod, "The efficacy and safety of the novel peripheral analgesic isovaline as an adjuvant to propofol for general anesthesia and conscious sedation: a proof-of-principle study in mice," Anesthesia \& Analgesia, vol. 121, no. 6, pp. 14811487, 2015.

[21] S. Katayama, M. Irifune, N. Kikuchi et al., "Increased $\gamma$ aminobutyric acid levels in mouse brain induce loss of righting reflex, but not immobility, in response to noxious stimulation," Anesthesia and Analgesia, vol. 104, no. 6, pp. 1422-1429, 2007.

[22] Y. Wu, N. Jia, C. Zhao et al., "Synergistic antinociception of propofol-alfentanil combination in mice," Pharmacology Biochemistry and Behavior, vol. 116, pp. 25-29, 2014.

[23] A. H. Lichtman, "The up-and-down method substantially reduces the number of animals required to determine antinociceptive ED50 values," Journal of Pharmacological and Toxicological Methods, vol. 40, no. 2, pp. 81-85, 1998.

[24] Z. Du, C. Wang, X. Tai, G. Wang, and X. Liu, "Optimization and characterization of biocompatible oil-in-water nanoemulsion for pesticide delivery," ACS Sustainable Chemistry \& Engineering, vol. 4, no. 3, pp. 983-991, 2016.

[25] R. Nau, F. Sörgel, and H. Eiffert, "Penetration of drugs through the blood-cerebrospinal fluid/blood-brain barrier for treatment of central nervous system infections," Clinical Microbiology Reviews, vol. 23, no. 4, pp. 858-883, 2010.

[26] Y. Shi, W. Porter, T. Merdan, and L. C. Li, "Recent advances in intravenous delivery of poorly water-soluble compounds," Expert Opinion on Drug Delivery, vol. 6, no. 12, pp. 1261-1282, 2009.
[27] C. G. Stäuble, R. B. Stäuble, S. J. Schaller, C. Unterbuchner, H. Fink, and M. Blobner, "Effects of single-shot and steady-state propofol anaesthesia on rocuronium dose-response relationship: a randomised trial," Acta Anaesthesiologica Scandinavica, vol. 59, no. 7, pp. 902-911, 2015.

[28] L.-H. Tan and N.-C. Hwang, "The effect of mixing lidocaine with propofol on the dose of propofol required for induction of anesthesia," Anesthesia \& Analgesia, vol. 97, no. 2, pp. 461-464, 2003.

[29] D. F. Driscoll, H. N. Bhargava, L. Li, R. H. Zaim, V. K. Babayan, and B. R. Bistrian, "Physicochemical stability of total nutrient admixtures," American Journal of Health-System Pharmacy, vol. 52, no. 6, pp. 623-634, 1995.

[30] S. Kaya, S. Turhanoglu, H. Karaman, S. Özgün, and N. Basak, "Lidocaine for prevention of propofol injection-induced pain: a prospective, randomized, double-blind, controlled study of the effect of duration of venous occlusion with a tourniquet in adults," Current Therapeutic Research-Clinical and Experimental, vol. 69, no. 1, pp. 29-35, 2008.

[31] Y. Masaki, M. Tanaka, and T. Nishikawa, "Physicochemical compatibility of Propofol-Lidocaine mixture," Anesthesia and Analgesia, vol. 97, no. 6, pp. 1646-1651, 2003.

[32] J. W. Park, E.-S. Park, S.-C. Chi, H. Y. Kil, and K.-H. Lee, “The effect of lidocaine on the globule size distribution of propofol emulsions," Anesthesia and Analgesia, vol. 97, no. 3, pp. 769-771, 2003.

[33] D. F. Driscoll, J. Nehne, H. Peterss, R. Franke, B. R. Bistrian, and W. Niemann, "The influence of medium-chain triglycerides on the stability of all-in-one formulations," International Journal of Pharmaceutics, vol. 240, no. 1-2, pp. 1-10, 2002.

[34] J. Han, S. S. Davis, and C. Washington, "Physical properties and stability of two emulsion formulations of propofol," International Journal of Pharmaceutics, vol. 215, no. 1-2, pp. 207-220, 2001.

[35] S. D. Roy and G. L. Flynn, "Solubility behavior of narcotic analgesics in aqueous media: solubilities and dissociation constants of morphine, fentanyl, and sufentanil," Pharmaceutical Research, vol. 6, no. 2, pp. 147-151, 1989.

[36] K. Kawakami, N. Oda, K. Miyoshi, T. Funaki, and Y. Ida, "Solubilization behavior of a poorly soluble drug under combined use of surfactants and cosolvents," European Journal of Pharmaceutical Sciences, vol. 28, no. 1-2, pp. 7-14, 2006.

[37] S. M. Berge, L. D. Bighley, and D. C. Monkhouse, "Pharmaceutical salts," Journal of Pharmaceutical Sciences, vol. 66, no. 1, pp. $1-19,1977$.

[38] A. Tsuji, "Small molecular drug transfer across the blood-brain barrier via carrier-mediated transport systems," NeuroRx, vol. 2, no. 1, pp. 54-62, 2005. 

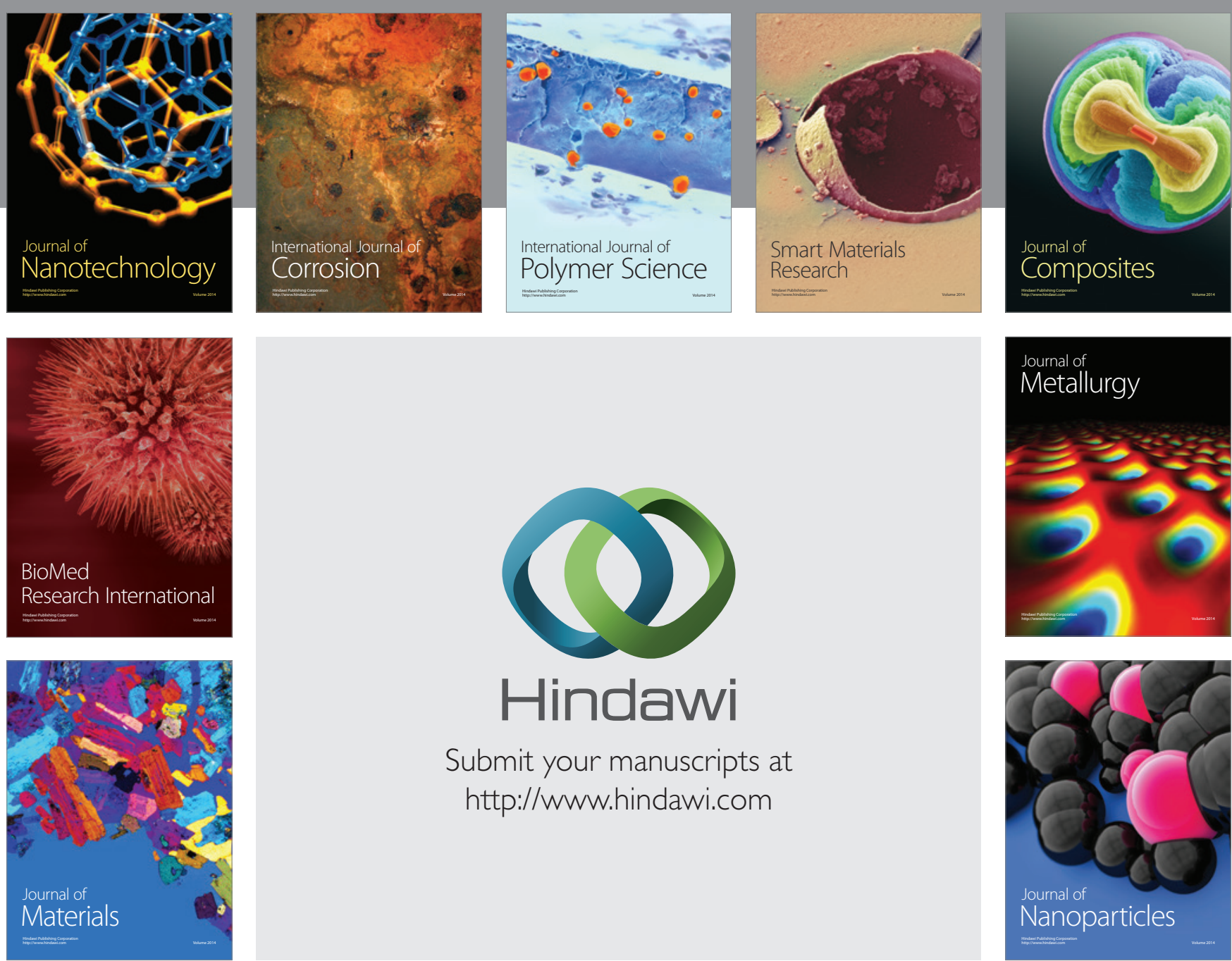

\section{Hindawi}

Submit your manuscripts at

http://www.hindawi.com

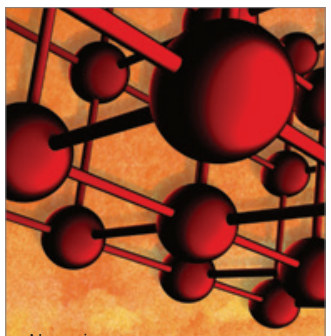

Materials Science and Engineering
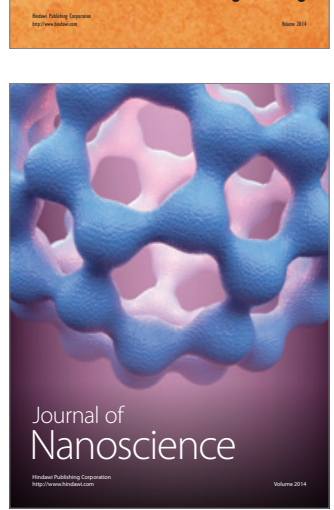
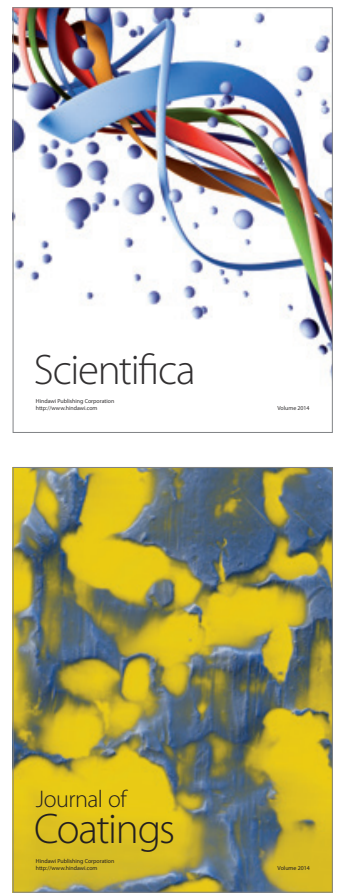
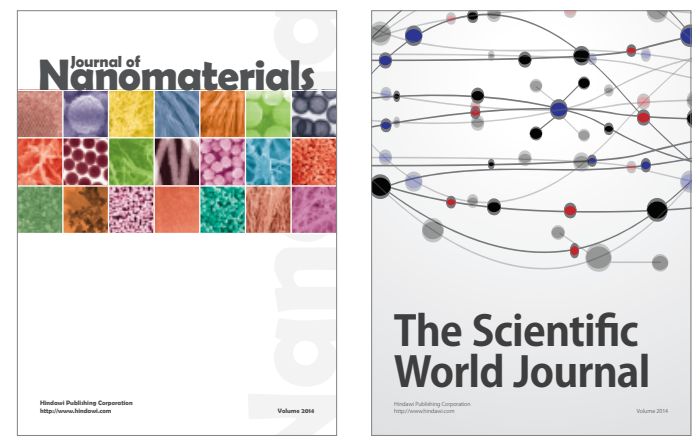

The Scientific World Journal
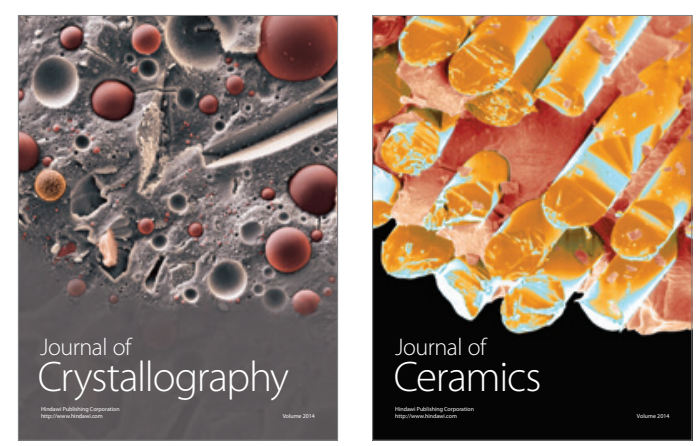
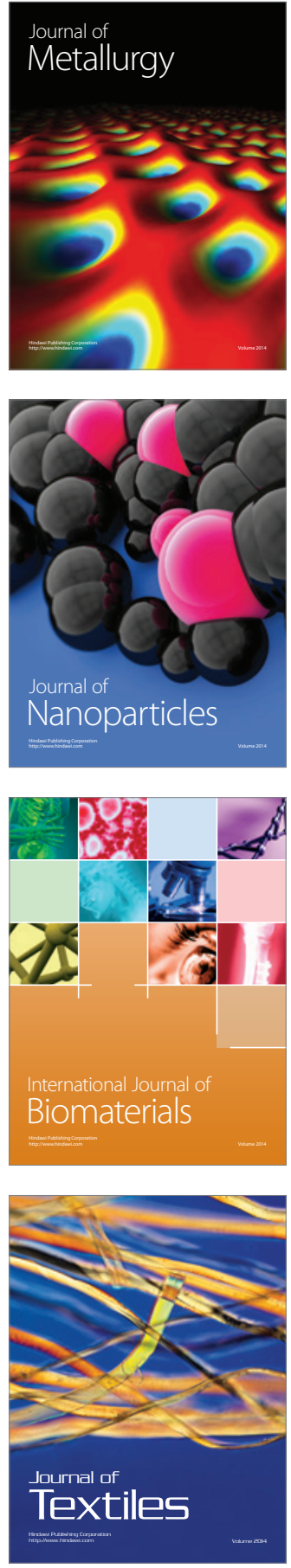